\title{
Chocano en la Revolución Mexicana
}

POr EMILIA ROMERO DE VALLE

José Santoş Chỏanio Hegó a México en agosto de 1912. La revolución encarnada en don Francisco I. Madero había triunfado en las eleceiones, designándolos Presidente de Méxi. co. Nadie preveía, por entonces, los sangrientos sucesos que habría de ocurrir pocos meses después.

En un libro que hace poco publicó en Santiágo de Chile la tercera esposa del poeta peruano, la costarricense Margarita Aguilar Mechado ( $\left.{ }^{1}\right)$, aparecen dos artículos de Chocano relacionados con aquella época. "Conoci y traté en México - dice- a los cinco hombres que, en tres períodos estrechamente eslabonados, desarrollaron en su totalidad la obra de la Revolución. El primer período, esto es, el inicial, pertenece solamente a Madero; el segundo período, esto es, el evolutivo, aparece entregado a la disputa entre Carranza y Villa; y el tercer período, esto es, el definitivo o culminante, corresponde en buena parte a Obregón, pero en mayor parte a Calles. Madero echa los cimientos sobre los que se va armando sólidamente, la maquinaria de la Revolución. Calles es quien

(1). Chocano. Sus últimos años. Santiago de Cliile. Arancibia Hnos. 1964. p.p.249. 
ajusta las últimas tuercas. La maquinaria puede ya funcionar sola; pero grandes aptitudes requieren la vigilancia de su funcionamiento..."( $\left.{ }^{2}\right)$.

Su primera entrevista con el Prusidente Madero tuvo lugar en el Alcázar de Chapultepec, a poco de su llegada al pais y fue presentado por el secretario Juan Sánchez Azcona, mediante gestiones del famoso orador Jesús Urueta. Aquella entrevista es de sumo interés porque Chocano - contradictorio toda su vida- si por un lado fue panegirista y amigo personal de Estrada Cabrera, el déspota guatemalteco, por el otro manifestó siempre grandísima adhesión por el Presidente Made. ro y sus propósitos de reforma política y social en México.

"Era él un hombre de pequña estatura -dice Chocano- ágil, nervioso, vibiante, con unos grandes ojos de mirada luminosa y envolvente, con una ancha frente de serenidad majestuosa; con una sonrisa ingenua y acariciadora $y$ con unos ademanes llenos de cfusión y cordialidad.

- He preferido recibirle aquí, para que conversemos paséandonos...

"En tal frase Madero me había desnudado su espíritu, enemigo de fórmulas, sencillo y franco, móvil y comunicativo... Púsose a mi lado, con las manos atrás, la frente levantada, riendo con lós ojos; y empezamos a platicar, mientras recorríamos con paso ligero fa espaciosa lerraza, con Urueta a un extremo y Sánchez Azconal álotró, reomo estudiantes cn fila que repasan en coro una lección, sintiendo los espíritus ensartados por el hilo de luz de una lírica fraternidad.

"Las primeras palabras de Madero fueron con respecto al Perú. Entre el Perú y México hay una corriente que pudiera llamarse "subhistórica", algo que en la psicología de los pueblos corresponde a los fenómenos subconscientes del psico-análisis de los individuos. Los imperios precolombinos, las Conquistas, los Virreinatos, fluyeron en nuestra plática hasta dejar sólo en pie el problema del indio.

-Es una raza dormida. Nuestro deber es despertarla. ¿Acaso nuestro indio no se parece hasta físicamente al ja-

(2). Obras completas, compiladas, anotadas y prologadas por Luis Alberto Sanclue\%, Mexico, Aguilar, 1954, pp. 1621; y Chocano. Sus últimos años, p. 110. 
"Al pronunciar estas palabras Madero se había detenido; y yo pude ver a sus espaldas, como formándole una decoración simbólica, el paisaje todo, digno del pincel de Velásquez, en que recortaban, sobre el viejo azul del fondo, sus perfiles enérgicos el Ixtaccíhuatl y el Popocatépetl.

"Miadero se me había presentado en su otro aspecto. Con la naturaleza a sus espaldas, aparecía iluminado al detenerse para decir esas palabras sencillas y profundas. Era el Apóstol..." ( $\left.{ }^{3}\right)$.

En estas frases puede notarse la admiración que sintió por Madero y la obra de transformación nacional que pretendía realizar en México. Su amistad hacia el político a quien consagraba con el título de "Apóstol" le valió ataques de los reaccionarios que por entonces aprovechaban la libertad absoluta de imprenta que la Revolución había establecido. Chocano fue atacado en "El Ahuizote" y en un número del 5 de octubre de 1912, apareció una caricatura en la cual se ve al poeta ensartado en las astas de un toro. Se hacía alusión, en esta caricatura, a una controversia que, sobre ideas políticas sostenía con el periodista Carlos Toro. Otro periodista que usaba el seudónimo de "Caifás" firmaba en el mismo periódico, en septiembre anterior unas "Cartas abiertas al eminentísimo poeta don José Santos Chocano", en las que se le insultaba y se hacía burla de él. Se le llamaba en ese diario "cantor oficial" a base de Edgard Poe y nróximo director del periódico político lmexicano (!) "La Raza" ${ }^{(4)}$.

Pero al lado dedestoscataqueso debidos a su adhesión a Madero y a la Revolución, tuvo amigos como el poeta Antonio Médiz Bolio, el ya mencionado orador Jesús Urueta y otros escritores y poetas que simpatizaban con los ideales maderistas. Al mismo tiempo dio recitales poéticos y conferencias en diversos teatros de la capital, entre ellos en el Arbeu.

No era Chocano un desconocido en los medios literarios de México, pues desde 1891 - cuando apenas contaba 16 años- sus poesías habían empezado a publicarse en diarios mexicanos y, poco después, en las mejores revistas literarias de entonces, como la "Revista Azul" (desde 1894), en "El Mundo" (desde 1895), en "Revista Moderna" (desde 1898) y "El Mundo Ilustrado" (Continuador de "El Mundo"), desde 1905.

(3). Obras completas, pp. 1621-1623; y Chocano. Sus últimos años, pp. 112-113.

Obras completas, po de mi Proceso, en Obras Completas, p. 1134, explica cómo fue cl proyecto de fundación de "La Raza". Según él fue una idea de don Gustavo Madero, quien propuso que se fundara ese diario durante un almuerzo al que asistieron, además de don Gustavo, el Vice-presidente Pino Suárez, y los Lics. Inés Novelo, Jesús Urueta y Antonio Médiz Bolio. Dicho proyecto no llegó a cristalizar. 
Chocano fue siempre supersticioso y en cierta forma un alucinado. No debe por esto extrañar las visitas que en compañia de Urueta y Médiz Bolio hacía a una anciana llamada Julita de Zamora, esposa de un coronel retirado de la época de la Intervención Francesa. Doña Julita - como dice que la llamaban todos- era una prodigiosa vidente y en una ocasión fue a visitarla con Médiz Bolio a fin de que les hablara sobre la situación política que por entonces se presentaba bastante difícil, por las amenazas de la reacción. La vidente les reveló que había visto a la ciudad de México bombardeada durante varios días y que Madero se hallaba envuelto en una nube sangrienta.

Los dos amigos resolvieron visitar al Vice-Presidente Pino Suárez a fin de exponerle sus temores y comunicarle lo que todos sabían en México: que se conspiraba contra el régimen en un bufete de abogado situado en las calles de Tacuba e Isabel la Cátólica y en la casa de un general, en Tactibaya. Pero -advierte Chocano en su articulo- "no hay que olvidar que Pino Suárez componía versos Y murió en poesía". Y al agradecerles la información les ctijo: "iGracias, amigos míos, gracias! Aprecio mucho la noble intensión de ustedes, pero estoy resuelto a dejar que se desarrollen los acontecimientos contra el Gobierno, sin esforzarme por impedirlos".

Y, añade Chocano: "Había en la voz de Pino un calor de la más honda sinceridad; yzomo notara en nuestros sem. blantes una angustiosa sorpresa, quiso explicarnos su actitud, descubriéndonos "unápágina histórica que hasta el momento actual es desconocida por el público del mismo México: "Amigo que merece casi tanta estimación como Uds. hizo saber los fundamentos de una determinación atribuida al General Victoriano Huerta para dar un golpe militar en próximo día. Puse tal hecho en conocimiento de mi colega el Ministro de la Guerra, a fin de que se hicieran las investigaciones y se tomasen las precauciones del caso. Me retiré esa noche a descansar de las fatigas diurnas, a mi hora acosiumbrada. Mi sueño fue violentamente interumpido por el ayuda de cámara que me hizo saber que el General Huerta me solicitaba con urgencia, esperándome en estos mismos salones en que estamos. Vi el reloj: eran las dos de la mañana. Me vestí presuroso y acudí a la llamacia, con la idea de que el General se había adelantado a dar el golpe militar anunciado y que venía a mí, después de haber ido, sin duda, por el Presidente Madero. Tal idea fue mayor en mí, en cuanto pude clistinguir al General con unifirme de gala. Avancé unos cuantos pasos 
hacia el General y le dije resignadamente: "-Aquí me tiene usted a sus órdenes". Entonces él, cuadrándose me replicó con energia: "-Quien está a sus órdenes, señor Vicepresidente de la República, es el General Victoriano Huerta, que ha venido a hacerle saber que él jamás manchará su hoja de servicios con una traición". Al escucharle sentí que me flaqueaban las piernas; y tuve ganas de arrodillarme y pedirle perdón. Desde ese momento estoy resuelto a que hagan de mí lo que quieran sin intentar yo defenderme".

"Al retirarnos Médiz Bolio y yo de la casa de Pino Suárez -continua Chocano- lo hicimos en un silencio tan profundo que sentíamos dormidos nuestros espíritus, con un sueño de que sólo, varios días después, nos vino a despertar el primer cañonazo que anunció el cumplimiento de la trágica profecía de la pitonisa de México" $\left({ }^{5}\right)$.

Ásesinados Madero y Pino Suárez el 22 de febrero de 1913, y posesionado Huerta de la Presidencia, Chocano inició su campaña contra éste. En "A.B.C." de Madrid y "El Dictamen" de Veracruz de esos días, aparecieron una relación y comentarios de cómo había triunfado "la usurpación" del General. Denunció que éste y Félix Díaz habían firmado el Pacto de la Ciudadela en el local de la Embajada de los Estados Unidos, el martes 18 de febrero, después de una reunión que duró desde las 9 de la noche hasta la 1 de la madrugada, cuando ya había sido apresado Madero. Yeel 11 de mayo de 1913, Chocano pronunció un discurso en el Hemiciclo de Juárez, en el que atacó violentamente a Huerta. El resultado fue que el Gobernador del D. F. General Samuel García Cuéllar ordenó su expulsión del país tildándolo de "extranjero pernicioso" y el poeta fue llevado por la policía a Veracruz para ser embarcado hacia La Habana $\left({ }^{6}\right)$.

En Cuba prosiguó sus ataques contra Huerta y Blanquet denunciando que en México habían instaurado una era de terrorismo. Y el \& de junio de 1913, según refire Luis Alberto Sánchez en su biografía del poeta $\left({ }^{7}\right)$, escribió un poema

(5). Chocano. Sus últimos años. pp. 125-128.

(6). "Santos Chocano y el agrarismo mexicano", por el Gral. Rubén García. "El Nacional", México, D.F. 21 junio, 1964.

(7) Aladíno o Vida y obra de José Santos Chocano, por L. A. Sánchez, México. Libro Míx. Elitorcs, 1960, p. 494-495. El poema aparece completo en "Obras Completas" de Chocano compiladas por el mismo Sánchez, México. Aguilar, 1954, p. 661, con la fecha Habana, 1914; pero en un viejo libro de recortes que perteneció a R.H.V. aparece fechado asi: "Golfo de México, 4 de Junio de 1913". 
titulado "Vida Náufraga" en el cual pide que se le entierre de pie en un metro cuadrado, deseo que se cumplió únicamente en 1965.

Busco obstinadamente sólo un metro cuadrado de tierra, en que los hombres me dejen levantar una torre muy alta, como nadie ha soñado y cuando, al fin, la encuentro, la tierra me hecha

(al mar!

Sólo un metro cuadrado busco de tierra firme... (Tal "el punto de apoyo" que pidió el Sabio aquel). Que si en él no la torre que soñé construirme, plantarían mis manos un rosal y un laurel.

Cuántas veces me empeño por poner a mi ensueño una base tranquila, cierta voz dice - iAndar! En vez de árbol que arraiga, soy apenas un leño condenado al capricho de las olas del mar... ${ }^{8}$ )

Este metro cuadrado que en la tierra he buscado, vendrá tarde a ser mío. Muerto, al fin, lo tendré. . Yo no pido ya ahora más que un metro cuadrado ${ }^{(8)}$ donde tengan un día que enterrarme de pié!

\section{EL REGRESO A MEXICO}

De Cuba ChocanbópasoaadPuerto Rico, siempre en plan de propaganda revolucionaria y de ataque a Victoriano Huerta. Pero en México los acontecimientos se precipitaban. Dor. Venustiano Carranza, Gobernador de Coahuila, se levantó contra el usurpador Huerta, proclamó el Plan de Guadalupe y se constituyó en jefe del Movimiento Constitucionalista. Fue secundado casi unánimemente por el pueblo y por algunos generales del Ejército Federal.

Vencedor Carranza, invitó a Chocano a que regresase a México y le acompañase en una jira por el Estado de Sinaloa. Durante esa jira dio varios recitales poéticos, entre ellos uno que tuvo lugar el 14 de febsero de 1914 en el Teatro Apolo de Culiacán, y en el que fue presentado por Lic. Isidro Fabela con estas palabras: "José Santos Chocano es un poeta y un apóstol; un poeta que pasará a la Historia de la Literatu.

(8). En Obras Completas este verso aparece asi: "condenado al insomnio convulsivo del mar..." El aqui citado correspondiente al viejo libro de recortes citado anteriormente. Por desgracia en ese libro no aparece el nombre de la revista en la que apareció. $Y$ en cuanto a la última estrofa, "Yo no pido ya" corresponde al libro viejo citado. En Obras Completas dice: "¡Yo no espero ya...!". 
ra de habla castellana como un representativo de nuestra América y de nuestra raza; y un apóstol, corolario del poeta que va predicando por el mundo una vida mejor, vigorosa y progresista para los países hispanoamericanos. El poeta que nació en el Perú es tan nuestro como si hubiese nacido en Tenochtitlán...( $\left(^{9}\right)$.

El 22 de febrero del mismo año, en que se commemoró el primer aniversario del absurdo asesinato de Madero y Pino Suárez, el poeta declamó en el Teatro Noriega de Hermosillo su "Sinfonía Heróica" en la que compara a los dos mártires con dos corderos y a Huerta con un monstruo:

Hay en los violines, mientras que se callan bronces y timbales, súplicas que llegan desde los confines, como si balaran; en la lejanía, corderos pascuales... Se ahueca en el bronce de largos clarines, viento de montañas; y surge un lamento, copiando en el viento un desgarramiento como de puñales que rompen

Un monstruo que acecha (Cuerpo de serpiente; cabeza redonda de tigre: tiene alas angulosas; ojos turbios y sesgados; un tajo en la

Hipócritamente, frente...)

corre resbalando sobre las alfombras de las palatinas ycisumtuosasvsalas». .

Día de traiciones, noche de tragedias. Horada el monstruo taimado; (las sombras y luego que Judas le presta sus galas Caín satisfecho se acuesta a su lado.

(Serpientes y tigres revuélvense dentro del vórtice airado,

que el monstruo prepara y abre de repente contra el buen Apóstol y contra su noble Discípulo

Y el monstruo enroscado

Amado). de anillo en anillo por fin se endereza; $y$, desde la altura, decídese y saca la horrible cabeza, cual gárgola echando por ávida boca copioso torrente de sangre, que sobre los tiempos futuros caerá gra(vemente...

(9). "Mis memorias de la Revolución", por Isidro Fabela. "Diario de Yucatán", Mérida, Yucatán. 21 marzo, 1956. 
Se ahueca en el bronce de largos clarines viento de montañas;

y surge un lamento, copiando en el viento

un desgarramiento como de puñales que rompen

entrañas...

En marzo siguiente Chocano fue a los Estados Unidos, y a bordo del barco que lo conducía escribió, el 2 de marzo de 1914 , una carta muy importante al poeta puertorriqueño Luis Llorens Torres. En esta carta revela sus deseos de revolucionar toda América y expresa su entusiasmo por Carranza, aunque después habría de variar de opiniones, adhiriéndose al grupo revolucionario de Francisco Villa.

Dice así: "Vuelvo descle la más honda entraña de la Revolución Mexicana. iContentísimo! Carranya es un Hombre de Estado. El más grande Hombre de Estado de la América Latina. Tal mi impresión sincera. ¿Ideas? ¿Planes? ¿Propósitos? Todos cuantos pudiéramos concertar entre Ud. y yo. Este hombre ha sido la más grata sorpressa de mi vida.

"Dentro de cuatro años estaré en Puerto Rico en el sentido que Ud. sabe. Ya ahora estoy seguro. Unión de Centroamérica. Confederación Antillana. República Federal Boliviana: todo se hará. Y los 500.000 soldados de México podrán organizarse ya hoy mismo

"Voy a estar siete đias cin Llà Habrnas đuego pasaré a Guatemala por otros siete días; luego, daré en New Orleans, New York y Washington, una serie de conferencias fundamentales sobre la Intervención Europea en México: "La organización de México como molde de las naciones hispanoamericanas" y "Las dos Américas dentro de diez años". ¡Usted verá! Detrás de cada palabra que yo diga, apuntará una bayoneta para sostenerla... ¡Voy a ser el Verbo! ¡Carranza será la acción! iAl fin! He sido más felíz que el Cínico, iya encontré a nuestro Hombre!... ( $\left.{ }^{10}\right)$.

En el artículo que transcribe en su libro Margarita Aguilar Machado a que hice referencia en párrafos anteriores, Chocano hace un paralelo entre Carranza y Villa, en la forma siguiente: "Carranza era un hombre solemne; Villa un atormentado. Carranza vivía en historia; Villa, en tragedia. Carranza llevaba una disciplina interior hasta el estoicismo; Villa sen-

(10). Antología de poetas contemporáneos de Puerto Rico, por Pedro Juan Labarthe. Mé xico, 1946 facsimilar entre pp. 15 y 17; y Obras Completas. pp.1027-1028. 
tíase indisciplinado hasta el desequilibrio nervioso colindante a la vez del genio y la locura. Carranza - justamente o noera inflexible, sin misericordia; Villa era un hombre que no tenía defectos sino solamente excesos.

“El paralelo de ambos hombres representativos es fácil de apreciar si se atiende a que Carranza tenía una sólida cultura histórica, a través de sus lecturas de César Cantú, que lo hacía preferentemente aparecer tratando de moverse con el ritmo de un Cónsul, Senador o Patriota romano y se atiende a que en Villa la inteligencia era tan extraordinaria como profunda la ignorancia, con lo que, careciendo de matices mentales, vivía en desequilibrio que lo hacía caer frecuentemente en el lado tenebroso...".

Y luego refiere cómo conoció al Primer Jefe:

"Conocí a Carranza en Hermosillo, en donde a la sazón ejercía la Primera Jefatura del Ejército Constitucionalista. Me recibió sentado detrás de la mesa escritorio en que atendía su despacho acompañado de sus jóvenes secretarios Espinosa Mireles y Alfredo Breceda. Al levantarse para estrechar mi mano, hízolo con cierta gravedad majestuosa, en la que yo recibí una impresión de autoridad que se hacía sentir sin gran esfuerzo.

"Como desde las primeras palabras yo llevé, naturalmente, el punto de vista de la Revolución Mexicana hacia los intereses continentales, Carranza se apresuró a manifestarme que el primer acto internacional de su gobierno sería el romper con el aislamiento en que México se había mantenido respecto de los demás países indoespañoles.

-Cubriré - me dijo, más o menos- la extensión de nuestra América con Legaciones y Consulados que, por primera vez, hagan sentir fraternalmente las ,palpitaciones del corazón de México en todo el Continente..." $\left.{ }^{11}\right)$.

Continúa Chocano describiendo sus relaciones con Carranza y dice que la última vez que estuvo con él fue en Durango, adonde llegó el poeta unos cuantos días después de que el Primer Jefe había hecho unas declaraciones sobre la política de Madero que habían disgustado a Francisco Villa y a su División del Norte.

(11). Chocano. Sus últimos años .. p. 116; Obras Completas, pp. 1623-1624. 
Carranza lo invitó a almorzar junto con el senador yucateco Magalloni y el periodista Trepiedi, quienes trataban de convencerlo de la conveniencia cle dar publicidad a un programa de principios de carácter económico-social; pero que Carranza no quiso escucharlos y al irse los interlocutores le preguntó a Chocano sus impresiones sobre aquella conversación. Chocano le contestó que habían salido disgustados y, entonces, Carranza se apresuró a decirle:

-Entonces no me han dicho la verdad.

-La verdad -le respondió Chocano- no llega nunca a los oídos de los que mandan.

Esta última frase, por lo que parece, impresionó al Primer Jefe y la repitió lentamente, como con amargura. Chocano le dijo entonces:

- ¿Quiere usted saber una? Un hombre de Estado necesita no sólo dominar, sino atyaer; y para ello resulta indispensable ser flexible.

-Yo necesitaría entonces nacer de nuevo- fue la respuesta de Carranza $\left.{ }^{12}\right)$.

CON FRANCISCO VILIA

A pesar de los elogios que Chocano había tributado a don Venustiano Carranza, al sobrevenir la ruptura del movimiento revolucionario, el poeta decidió seguir a la fracción encabezada por el General Francisco Villa. Y se le pucde ver, así, frecuentando el trato del guerrillero y declamando en el campamento villista, en forma que entusiasmaba a aquellas huestes.

E1 Lic. José Vasconcelos, aunque en 1925 atacó a Lugones y a Chocano en su famoso artículo "Poetas y Bufones", tuvo una vez palabras de elogio para el poeta peruano al incitar a los de México a escribir grandes poemas, algo así como "el poema cósmico": "Allí tienen ustedes, por ejemplo - dijoel único que se ha atrevido a hacer en nuestra América el poema de aliento es Chocano. Cuando yo lo oía en el campamento villista recitar sus versos y notábamos el efecto que producía

(12). Id. Id. pp. 117-118. y Obras Completas, pp. 1624. 
en las chusmas, Chocano me parecía algo así como Homero" $\left({ }^{13}\right)$.

Ya hemos visto en párrafos anteriores, el paralelo que Chocano trazó entre Carranza y Villa. Vale la pena reproducir la siguiente estampa del famoso guerrillero que se publicó en Santiago de Chile en 1933 y en San José de Costa Rica en 1938, $\mathrm{v}$ que ha sido reproducida por Luis Alberto Sánchez en las Obras Completas del poeta y por Margarita Aguilar Machado en su libro ya mencionado: Chocano. Sus tiltimos años.

"Luminoso y sombrío, él era siempre grande. El día que le conozco me brinda con licores, pero el bebe agua clara. Le ofrezco un cigarrillo y me da las gracias, pero no acepta.

- Cómo - le interrogo- ¿es posible que no le gusten ni el licor ni el tabaco?.

Me responde sonriendo: sobrio como él.

- He pasado veinte años de mi vida en el desierto y soy

"Tal fràse no cra sólo hermosa sino verdadera. Pancho Villa no tuvo sino dos obsesiones: poseer la hembra y matar al enemigo. Sobrio como el desierto, sólo se sentía atraído por el Amor y por la Muerte.

"Cierta mañana que concél desayuno, me invita a conversar de asuntos interesantes en su despacho de Chihuahua, en donde por entonces se ejecutaban diariamente muchos fusilamientos. De pronto aparecen en grupo a la puerta del despacho los que tenía él designados como ejecutores de tales fusilamientos diarios. El Jefe de ellos se adelanta, interrumpiendo nuestra conversación con esta demanda:

-iOrdenes, mi general!

Pancho Villa le grita:

— ¡Hoy no hay carne! ¡Váyanse, que no hay carne!.

"Y con majestad de domador que sacude del asedio a sus fieras, sigue tranquilamente conversando conmigo. Yo sonrío,

(13). "Vasconcelos-Chocano" por Rafael Heliodoro Valle, "Excelsior", México D. F. 2 diciembre 1925; "Repertorio Americano", San José de Costa Rica, 18 enero 1926;

"La Hoguera", Lima, 21 abril 1926. 
pensando en que la frase es cligna de una página de Suetonio ..." $\left({ }^{14}\right)$.

Villa le encargó enionces ir a los Estados Unidos a fin de comprar armas para ia División del Norte y hacer propaganda en su favor. En los primeros meses de 1915, Chocano fue a El Paso acompañado y protegido por algunos "dorados" de Villa. Por ese tiempo publicó en San Antonio el folleto que tituló: "Interpretación Sumaria del Programa de la Revolución Mexicana", precediclo por una carta de Villa que dice así: "Torreón, Coahuila, 29 de marzo de 1915/. Señor D. José Santos Chocano. Presente/. Muy estimado amigo: Complázcome en felicitar a Ud. por haber sabido interpretar los ideales de la Revolución Mexicana. Los Principios del Programa en (la forma en) que los ha interpretado, se ajustan, por completo, a las aspiraciones y necesidades del Pueblo de que me honro en ser celoso defensor.- Como habrá Ud. podido apreciarlo personalmente en su calidad de testigo de excepción, esos Principios han comenzado a ser puestos en práctica en la zona dominada por las fuerzas de mi mando/. La labor de Ud. acompañando al Pueblo Mexicano en estas horas de dura prueba, es digna del mayor encomio . Créame su amigo affmo. y muy s. s. (Firmado). Francisco Villa, General en Jefe de las Operaciones Militares".

En este "Programa" que tanto gustó a Villa, Chocano presentaba una síntesis ide los problemas cue se pretendía atacar y resolver: "Problema Agrario, Problema Bancario, Problema Minero, Problemar Contilibutivo, Problema Municipal, Problema Obrero, Problema Pedagógico, Problema Militar, Problema Sanitario, Problema Usurario, Problema Editorial, Problema Judicial, Problema Legislativo, Problema Electoral". Y al final aparecen largas consideraciones acerca de "El carácter agrario de la Revolución Mexicana" (15).

De este viaje a El Paso en 1915, un mexicano llamado A. Diez Gutiérrez ha referido un incidente muy divertido, con el título siguiente: "Sí. Esto ha pasado. Santos Chocano en aprietos". Según Diez Gutiérrez, al llegar el poeta a esa población, el "Paso Morning Times", anunció que se celebraría un mitin durante el cual Chocano dirigiría la palabra al pueblo. Por entonces El Paso se hallaba repleto de mexicanos anti-revolucio-

(14). "Ariel", San José de Costa Rica, 1ro. Julio 1938, serie VII (21): 577. Una variante - mejor dicho la misma versión antes de ser corregida como apareció en "Ariel y que es la que aquí reproduzco- apareció en "La Nación", Santiago de Chile, 12 noviembre 1933, que reproduce Margarita Aguilar Machado' en Chocano.

(15). Obras Completas... pp. 118-120; y L. A, Sánchez en Obras Completas, p. 1625. 
narios desterrados que esperaban el momento oportuno para regresar a su país. No es, pues, de extrañar que el teatro en donde se celebró el mitin estuviera pletórico de espectadores. Chocano se presentó como tenía costumbre, vestido impecablemente de frac. Su discurso se inició con un ataque contra $\mathrm{Ca}$ rranza: "México - dijo- está aherrojado por la barbarie carrancista". Al escuchar estas palabras, los aplausos resonaron en todo el teatro. Pero enseguida empezó a elogiar a Villa diciendo que tenía madera de héroe y que "se había reencarnado en él el espíritu de Morelos". tario:

Al escuchar esto, una voz anónima le gritó descle el luneza?

— ¿Cuántos bilimbiques te pagaron por esto, sinvergüen-

Chocano no se inmutó en lo mínimo y replicó altanero:

- No hay en todo México oro suficiente para pagar una sola de mis palabras $\left({ }^{16}\right)$.

En El Libro de mi proceso, Chocano dice que Villa confió a su "corrección y desinterés" giros por valor de 60,000 dólares para comprar municiones, y pone de testigos a don Evaristo y don Alberto Madero, de la forma honorable con que procedió $\left({ }^{17}\right)$.

Chocano ya nōoregresóca México y años después, en Oro de Indias, apareció el poema "Ultima rebelión" (L. A. Sánchez en Obras Completas le asigna el año de 1920, como fecha de composición, sin seguridad), dedicado "A Francisco Villa, el Flamígero" y en que impreca al guerrillero en esta forma:

"Caes... Caes... ¡No importa, bandolero divino! (Remo, Rómulo: el crimen es a veces ritual...) Cierta voz, como a Pablo, te llamó al buen camino; pero ¿quién te diría: - Piensa bien y obra mal?...

Un demonio y un ángel, en tremendas porfías disputáronse el signo de tu oculta intención; y es así cómo, a solas, sin querer, sentirías el trajín de cuatro alas dentro del corazón...

(16). "Ultimas Noticias de Excelsior", 2a. edición, México D. F. 22 y 23 abril 1946, p. 3.

(17). Obras Completas... pp. 1135. 
Loco die gloria, hiciste tal vez aprendizaje de tues desorbitadas artes, en la lección que te enseñó, alumbrando tu espíritu salvaje, Hércules asesino, y Mercurio ladrón...

Por entre el laurel trágico en que ciñes tu sienes, la locura ha revuelto su pavorosa crin: tan grande en la batalla como en el crimen, tienes lista, al puñal, la diestra y el oído al clarín.

IIijo de águila y tigre, sientes en las entrañas yo no sé qué delirio de metal en crisoi: agua pura que gime bajo negras montañas 0 arrebol salpicado con la sangre del Sol.

¡Sábelo: tu Fortuna se siente fatigada... Sábelo: ya su entrada te cierra el porvenir... $\mathrm{Y}$, pertinaz, te obstinas en esgrimir tu espada como ave que sacude sus alas al morir!

Zigzagueando en el aire, caes con la caída que en las sombras eternas desenvuclve Luzbel. Caes... caes, mirando con desprecio la vida y a la vez sujetándote a la frente el laurel.

¡En la caída, a veces resistirte procuras: justo es que, al caer, vuelvas los ojos hacia atrás; que cuando se desprende porcfin de las alturas el quejse siente crayol tiene que hacer zigzás.

Finalmente, podemos preguntarnos qué fue lo que inclujo a Chocano a tomar partido, en la forma en que lo hizo, en un asunto enteramente mexicano, como fue la Revolución, siendo él un extranjero. Para eso hay que conocer el carácter ególatra de Chocano que ansiaba en todas partes ocupar un lugar destacado y se llamaba "el cantor de América". Por eso no hay que extrañar que también se auto-titulara "el Verbo de la Revolución" y pensara, como lo dice en la carta dirigida a Llorens Torres, que Carranza sería la Acción.

En la "Introducción" al "Programa de la Revolución Mexicana", que ya cité y que se publicó en El Paso, Texas, el 25 de abril de 1915, explica su pensamiento y el por qué de su intromisión en política ajena a su propio país. Dice que la Revolución Mexicana tiene "una importancia excepcional en la Historia de los grandes movimientos sociales y políticos de 
nuestros Tiempos". Opina, luego, que la Revolución Inglesa estableció la Libertad Política, la Francesa, la Igualdad Social y que "la Revolución Mexicana vendrá a ser, históricamente, la Gran Revolución de América - y quiere hacer la fraternidad económica de los hombres" $\left({ }^{18}\right)$.

Continuando su explicación dice que: “Tres grandes movimientos armados le han abierto a México la senda de su destino manifiesto: el de Independencia, el de la Reforma y éste que habrá de ser el de la Organización. En el primero, que se consolidó con la caída del Imperio de Iturbide, colaboró eficazmente un ilustre ecuatoriano, don Vicente Rocafuerte, que después de servirle como agente en los Estados Unidos de América, firmó como el Ministro suyo en Inglaterra, la primera Convención Internacional que se celebrara en México.

"En el segundo colaboró eficazmente un intelectual cubano: don Pedro Santacilia, que alcanzó la suerte de ser secretario particular del Benemérito de las Américas", don Benito Juárez. El ejemplo de alto hispanoamericanismo de don Vicente Rocafuerte y don Pedro Santacilia, me ha estimulado a colaborar en la actual Revolución Mexicana, si no con eficacia, con toda voluntad, previendo que, después de este gran movimiento va a quedar francamente abierto, para toda nuestra América intertropical, el período de la verdadera Organización" $\left({ }^{19}\right)$.

Así, pues, segun chocano, fueron estasarazones las que lo indujeron a tomar parte en el movimiento revolucionario mexicano de 1910.

México. D. F. septlembre de 1966.

(18). Obras Completas... pp. 1029; y Chocano. Sus últimos años... pp. 131-134 (19). Id. Id. 